\title{
The Cause of Tension in the Measurements of the Hubble Constant
}

\author{
Naser Mostaghel \\ Department of Civil Engineering, University of Toledo, Toledo, USA
}

Email address:

naser.mostaghel@utoledo.edu

\section{To cite this article:}

Naser Mostaghel. The Cause of Tension in the Measurements of the Hubble Constant. American Journal of Astronomy and Astrophysics. Vol. 8, No. 4, 2020, pp. 80-86. doi: 10.11648/j.ajaa.20200804.13

Received: October 16, 2020; Accepted: November 6, 2020; Published: December 8, 2020

\begin{abstract}
The latest reported measurements for the evaluation of the Hubble constant by two different teams, the Riess et al. (2019) in USA and the Plank Collaboration (2018) in Europe, in spite of increased accuracy of measurements, have resulted in significantly different values. This tension between the results of the two different measurement methodologies has been a vexing puzzle. To resolve this tension we present a two-parameter kinematic model which predicts two different values for the Hubble constant. Each predicted value is essentially identical to one of the measured values. The two parameters are the redshift and the age of the universe. Using the model we show that the elapsed time between the event of the Big Bang and the event of the release of photons, at the decoupling time, is the factor causing the tension in the above two measurements of the Hubble constant. This model also yields a simple relation for predictions of distance moduli. It is shown that the predicted distance moduli are remarkably consistent both with the observational data and with those of the standard Lambda CDM model. As the predicted values of the Hubble constant are essentially identical to the corresponding measured values, it is concluded that the difference in the measured values of the Hubble constant is due to how the elapsed time, between the event of the big bang and the event of the appearance of photons, has incorporated itself into the measurement methodologies.
\end{abstract}

Keywords: Cosmic Expansion Rate, Hubble Constant, Redshift, Distance Modulus, Dark Energy

\section{Introduction}

Although the standard $\Lambda$ CDM has been successful in addressing many cosmological problems, it has also given rise to major theoretical issues [1]. For example there is no universal agreement on the issues of cosmological constant and dark mass/energy. Also, in spite of increased accuracy of measurements, there are significant differences in the measured value of the Hubble constant, $H_{0}$. Reiss et al. [2] reports a measured value of $H_{0}=74.03 \pm 1.42 \mathrm{~km} \mathrm{~s}^{-1} \mathrm{Mpc}^{-1}$, while the value reported by the Plank Collaboration [3] is $H_{0}=67.66 \pm$ $0.42 \mathrm{~km} \mathrm{~s}^{-1} \mathrm{Mpc}^{-1}$. It has not been possible to explain the cause of the divergence of the above two measured values via the $\Lambda$ CDM model. A very recent approach by Freedman et al. [4] estimates a value for $H_{0}$ which is midway between the above two values. However the Freedman approach does not explain the cause of tension between the above two measured values of the Hubble constant. But the assumptions of isotropy and homogeneity imply a unique expansion rate at the present time. Consequently one concludes that there must be some quantity whose treatment has caused the difference in the above two measurements of $H_{0}$.

Since the Hubble constant itself, as a parameter, is intimately involved in the determination of the energy density parameters in the $\Lambda$ CDM model, to explain the cause of tension in the measured values of $H_{0}$, one needs to develop a different model. The new model should not involve the Hubble constant as one of its parameters, and it should be able to demonstrate the cause of tension in the above two measured values of $H_{0}$. Clearly the predictions of the new model must also be consistent with both the observational data and the standard $\Lambda$ CDM model. As a new model, we propose an analytical kinematic model with two parameters. The two parameters are the age of the universe and the redshift. The proposed model, being a kinematic model, does not explicitly involve any kind of mass and/or energy, dark or otherwise; consequently it is free of the cosmological 
constraints associated with $\Lambda$ CDM-based models.

Through the proposed analytical model we find two values for the Hubble constant: $H_{0}=67.860 \mathrm{~km} \mathrm{~s}^{-1} \mathrm{Mpc}^{-1}$ and $H_{0}=73.848 \mathrm{~km} \mathrm{~s}^{-1} \mathrm{Mpc}^{-1}$. These predicted values of $H_{0}$ are essentially identical to the corresponding values estimated by Reiss et al. and by the Plank Collaboration respectively. It will be shown that the difference between the above two measured values for $H_{0}$ is caused by the manner in which the elapsed time, between the event of the big bang and the event of appearance of photons, has incorporated itself in the measurement methodologies. This work aims to develop a model to explain the cause of tension in the aforementioned measurements and to validate the model through comparisons of its prediction of distance moduli with both observational data and the standard $\Lambda$ CDM model.

Next, in section 2, we develop a relation for the evaluation of the luminosity distance. This relation involves an unknown constant $v$ which is evaluated in section 3 . In section 4 we present the process through which the model predicts two values for $H_{0}$. In sections 5 and 6 we demonstrate the remarkable consistency of the predictions of the model with the observational data and with the standard $\Lambda \mathrm{CDM}$ model. The conclusions and remarks are presented in section 7 .

\section{Evaluation of the Luminosity Distance and Expansion Acceleration}

Throughout this model we assume the universe to be flat, isotropic and homogeneous. Further we assume the age of the universe, that is, the time elapsed between the event of the big bang and the present time, to be 13.8 giga years. To develop a relation between the redshift and time we consider an inertial acceleration represented by

$$
A_{i}=\frac{V^{2}}{c t}=\frac{(c \zeta)^{2}}{c t}=\frac{c \zeta^{2}}{t}
$$

where $A_{i}$ represents the inertial acceleration; $V=c \zeta$ represents a velocity; $t$ designates time; c represents the speed of light; and $\zeta$ represents the redshift. It should be noted that $V$ does not represent the expansion velocity. The expression for the expansion velocity is developed in section 4. Multiplying both sides of the above relation by $t^{2} / c$ yields

$$
\frac{t^{2}}{c} A_{i}=\zeta^{2} t
$$

To avoid the singularity at $t=0$, we consider the radius of the universe to have been equal to $c t_{p}$ at the instant of the Big Bang, where $t_{p}=5.39116 \times 10^{-44}$ is the Plank time in seconds [5] and $\zeta_{p}$ represents the corresponding redshift, which we will calculate later on; because it corresponds to Plank time we designate $\zeta_{p}$ Plank redshift. Since any reduction in time is associated with a corresponding increase in redshift, considering equation (2) and the instant of the Big Bang, we let

$$
\frac{t^{2}}{c} A_{i}=(\zeta(t))^{2} t=\zeta_{p}^{2} t_{p}=\text { Constant }
$$

It is noted that the above relation is a conjecture. Its validity will be shown through consistency of the predictions of the model with the observational data and with the standard $\Lambda$ CDM model. Based on relations (3) one obtains the following relations:

$$
\begin{array}{r}
(\zeta(t))^{2} t=\left(\zeta_{p}\right)^{2} t_{p} \\
\zeta(t)=\sqrt{\frac{\left(\zeta_{p}\right)^{2} t_{p}}{t}} \\
\zeta\left(t_{0}\right)=\sqrt{\frac{\left(\zeta_{p}\right)^{2} t_{p}}{t_{0}}}
\end{array}
$$

where $t_{0}=13.8$ giga years represents the age of the universe at the present time. It is noted that $\zeta_{0}=\zeta\left(t_{0}\right) \neq 0$. The observational redshift, $z$, is defined by

$$
Z=\frac{\zeta}{\zeta_{0}}-1=\frac{\sqrt{\frac{(\zeta p)^{2} t_{p}}{t}}}{\sqrt{\frac{\left(\zeta_{p}\right)^{2} t_{p}}{t_{0}}}}-1=\sqrt{\frac{t_{0}}{t}}-1
$$

Clearly at $t=t_{0}$ the above relation yields $z\left(t_{0}\right)=0$. The relation between the scale factor, a, and the redshift, $z$, is given $[6,7]$ by

$$
a=\frac{1}{1+z}
$$

To avoid the use of the Hubble constant, here we represent the luminosity distance [6-8], for a flat universe, by

$$
d_{L}=2 c t_{0} k(1+z) a
$$

In order to satisfy the condition $d_{L}=c t_{0}$ at $z=0$ and also to satisfy the assumed isotropy, we replace the term $z$, in the above relation, by $(z-0.5)$ for the luminosity distance. Thus

$$
d_{L}=2 c t_{0} k(1+(z-0.5)) a=c t_{0} k(1+2 z) a
$$

Here the factor $k$ is represented by

$$
k=(1+z)^{v}
$$

where $v$ is a constant whose value will be calculated in the next section. Considering the above two relations, the luminosity distance is given by

$$
d_{L}=c t_{0}(1+z)^{v}(1+2 z) a
$$

Substitution for the scale factor, $a$, from equation (8) into the above equation yields the luminosity distance as a function of redshift as

$$
d_{L}=c t_{0}(1+z)^{(-1+v)}(1+2 z)
$$

The validity of this relation is discussed in sections 5 and 6 . Substitution for $\mathrm{z}$ from equation (7) into the above relation yields the luminosity distance as a function of time as

$$
d_{L}=c t_{0}\left(\frac{t_{0}}{t}\right)^{0.5(-1+v)}\left(-1+2 \sqrt{\frac{t_{0}}{t}}\right)
$$


Next, the expansion acceleration, $A_{e}$, is given by the double differentiation of the luminosity distance, given by equation (14), as

$$
A_{e}(t)=\frac{d^{2}}{d t^{2}} d_{L}=\frac{c}{t_{0}} \frac{\left(\frac{t_{0}}{t}\right)^{v / 2}\left(4 v+2 v^{2}+\sqrt{\frac{t}{t_{0}}}\left(1-v^{2}\right)\right)}{4\left(\frac{t}{t_{0}}\right)^{2}}
$$

Therefore at the present time, $t=t_{0}$, the above equation yields the present-time value of the expansion acceleration as

$$
A_{e}\left(z_{\mathrm{H}}\right)=\frac{c}{t_{0}} \frac{\left(1+z_{\mathrm{H}}\right)^{4+v}\left(4 v+2 v^{2}+\frac{1}{\left(1+z_{\mathrm{H}}\right)}\left(1-v^{2}\right)\right)}{4}
$$

\section{Evaluation of the Constant $v$}

It is noted that, according to relation (7), at $t=t_{0}$ one has $z\left(t_{0}\right)=0$. This implies that the expansion acceleration in equation (16) is based on $z\left(t_{0}\right)=0$. Because of the assumed homogeneity and isotropy properties, at any instant of time, including the instant of the present time, the expansion acceleration is considered to be the same for all values of the redshift, including the redshift at the horizon (at the boundary of the observable universe).

We calculate the numerical value of the constant, $v$, by equating the present-time value of the expansion acceleration at redshift, $z\left(t_{0}\right)=0$, and the present-time value of the expansion acceleration for the redshift at the horizon, $z_{H}$.

To find a relation for evaluating the present time value for the expansion acceleration at $z=z_{H}$ we consider relation (3). Based on relation (3) the inertial acceleration, $A_{i}(t)$, can be represented by

$$
\zeta_{p}=\frac{\lambda_{o}}{\lambda_{e}}-1=\frac{\tau_{\text {then }}}{\tau_{\text {now }}}-1=\frac{\tau_{p}}{2.72548}-1=
$$

where $\lambda_{o}$ and $\lambda_{e}$ are the observed and emitted wavelengths. From the CMB radiations at the time of decoupling, the temperature at the horizon has been estimated to have been about $\tau_{\text {then }}=3000^{\circ} \mathrm{K}$, and the black body radiation temperature now is $\tau_{\text {now }}=2.72548^{\circ} \mathrm{K}$ [11]. Proceeding as in relations (20) we obtain the value of the redshift at the horizon as

$$
z_{H}=\frac{\tau_{\text {then }}}{\tau_{\text {now }}}-1=\frac{3000}{2.72548}-1=1099.72
$$

The Plank time is $t_{p}=5.39116 \times 10^{-44} \mathrm{~s}$. Assuming the present time age of the universe, $t_{0}$, to be 13.8 giga years, substituting for $\zeta_{p}$ and $z_{H}$ from relations (20) and (21) into equation (19), the numerical solution of equation (19) yields the value for the constant $v$ as

$$
v=0.0845134
$$

Both sides of equation (19) are plotted in figure 1. The intersection of the two curves in this figure also shows the solution for the constant $v$ as presented above.

$$
A_{i}(t)=\frac{c}{\mathrm{t}} \frac{t_{p}}{\mathrm{t}} \zeta_{p}^{2}
$$

Using heuristics, we transform the inertial acceleration at the present time, $A_{i}\left(t_{0}\right)$, in the above equation, via the factor, $2\left(1+z_{H}\right)^{-(1+v)}$ to the following relation to represent the expansion acceleration at the present time, $\tilde{A}_{e}\left(t_{0}\right)$, at $z=z_{H}$. That is

$$
\tilde{A}_{e}\left(z_{\mathrm{H}}\right)=\frac{c}{t_{0}} \frac{1}{2}\left(1+z_{H}\right) \frac{t_{p}}{t_{\mathrm{H}}} \zeta_{p}^{2}
$$

where the factor 2 accounts for the isotropic expansion starting at the Big Bang. It can be shown that the factor $\left(1+z_{H}\right)^{-(1+v)}$ corresponds to the principal part of the second fractional derivative (the fraction is $v+1 / 2$ ) with respect to redshift, $z$, (at $z=z_{H}$ ) of the factor $k$ which was introduced in equation (9) and defined by equation (11). At the present time the expansion acceleration at $z=0$ is equal to the expansion acceleration at $z=z_{H}$. Therefore $A_{e}\left(t_{0}\right)$ in equation (16) is equal to $\tilde{A}_{e}\left(t_{0}\right)$ in the above equation. That is $A_{e}\left(t_{0}\right)=\tilde{A}_{e}\left(t_{0}\right)$. Thus

$$
\frac{\left(1+z_{\mathrm{H}}\right)^{4+v}\left(4 v+2 v^{2}+\frac{1}{\left(1+z_{\mathrm{H}}\right)}\left(1-v^{2}\right)\right)}{4}=\frac{1}{2}\left(1+z_{H}\right) \frac{t_{p}}{t_{\mathrm{H}}} \zeta_{p}^{2}
$$

To solve the above equation for $v$, we need the values of the Plank redshift, $\zeta_{p}$, and the redshift at the horizon, $Z_{H}$.

In the following the Plank redshift, $\zeta_{p}$, is evaluated based on the Plank temperature, $\tau_{p}=1.416807993748161 \times$ $10^{32} \mathrm{~K}$, based on the black body temperature at the present time, and based on Wien's displacement law [8-10] according to the following relations.

$$
\frac{1.41681 \times 10^{32}}{2.72548}=5.19838 \times 10^{31}
$$

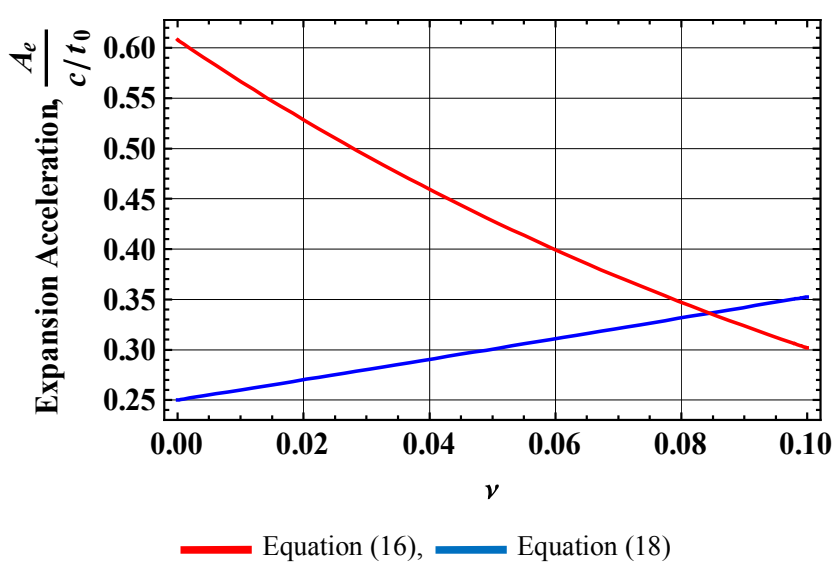

Figure 1. Variations of Expansion Acceleration with the Constant $v$.

\section{The Cause of Tension in the Measurements of the Hubble Constant}

The proposed model involves neither the Hubble constant nor dark matter/energy, but we use this model to analytically predict two different values for the Hubble constant. Considering the expression for the luminosity distance given 
in equation (14), the expansion velocity is given by

$$
V_{e}(t)=-2 \frac{d}{d t} d_{L}=\frac{c\left(\frac{t_{0}}{t}\right)^{v / 2}\left(\sqrt{\frac{t_{0}}{t}}(1-v)+2 v\right)}{\frac{t_{0}}{t}}
$$

The minus sign in the above equation takes care of the fact that observing from earth, the direction of increasing expansion rate is opposite to the direction of increasing time. And the factor 2 is to satisfy the assumed isotropy. At the present time $t=t_{0}$, the above equation yields the presenttime value of the expansion velocity as

$$
V_{e}\left(t_{0}\right) / c=1+v
$$

Since the expansion velocity at the present time must not exceed $c$, the extra velocity, $v$, in the expansion velocity, $V_{e}\left(t_{0}\right) / c$, in the above equation, is presumed to have been caused by changes of light's travel time. Here using the model we first find the changes in the light's travel time and then show how the model predicts two values for the Hubble constant.

The isotropic expansion implies that the velocity change, $v$, along the line of sight is composed of two velocities each equal to $v / 2$ but in opposite directions to each other. That is, the relative total velocity change is equal to $v$. But now one velocity change is $v / 2$ while the other is $-v / 2$. This yields the following two values for the non-dimensioned expansion velocity at the present time:

$$
V_{e}\left(t_{0}\right) / c=1 \pm \frac{v}{2}
$$

We find the elapsed time, $\delta t_{0}$, between the events of the Big Bang and the appearance of photons through the following relation

$$
\left(1 \pm \frac{v}{2}\right)\left(t_{0}+\delta t_{0}\right)=t_{0}
$$

Substitutions of the value of $v$ as given in equation (22) and the age of the universe $t_{0}=13.8$ giga year $=$ $4.35495 \times 10^{17} s$ into the above equation yield

$$
\begin{aligned}
& \delta t_{01}=\frac{-(v / 2) t_{0}}{1+(v / 2)}=-1.765639206540806 \times 10^{16} s \\
& \delta t_{02}=\frac{(v / 2) t_{0}}{1-(v / 2)}=+1.921442369143724 \times 10^{16} s
\end{aligned}
$$

Now the Hubble times, $t_{H_{0}}$ and $t_{H_{02}}$ are evaluated through substitutions of the above values in the following relations

$$
\begin{gathered}
t_{H_{01}}=\frac{1}{H_{01}}=t_{0}+\delta t_{01}=4.178384879345919 \times 10^{17} \mathrm{~s} \\
t_{H_{02}}=\frac{1}{H_{02}}=t_{0}+\delta t_{02}=4.547093036914372 \times 10^{17} \mathrm{~s}
\end{gathered}
$$

Considering

the

fact that Megaparsec $=3.08567758149137 \times 10^{22} \mathrm{~m}$, then the above relations yield

$$
\left(H_{01}\right)_{M p c}=\frac{1}{t_{H_{01}}} \times \frac{\text { Megaparsec }}{1000}=73.848 \mathrm{~km} \mathrm{~s}^{-1} \mathrm{Mpc}^{-1}
$$

$$
\left(H_{02}\right)_{M p c}=\frac{1}{t_{H_{02}}} \times \frac{\text { Megaparsec }}{1000}=67.860 \mathrm{~km} \mathrm{~s}^{-1} \mathrm{Mpc}^{-1}
$$

It is seen that the value given by equation (31) is consistent with the $H_{0}=74.03 \pm 1.42$ obtained by Reiss et al. [2]. As well, the value given by equation (32) is consistent with the $H_{0}=67.66 \pm 0.42$ obtained by the Plank Collaboration [3].

It is clear that in the evaluation of the Hubble time, $t_{H_{01}}$ as given by equation (29), the light travel time is reduced by the elapsed time, $\delta t_{01}$, given by equation (27). As the value of $H_{01}$ given by equation (31) is consistent with the value of $H_{0}=74.03 \pm 1.42$ obtained by Reiss et al. [2], it is concluded that the Reiss et al. methodology of estimating the Hubble constant effectively results in a subtraction of the elapsed time, $\delta t_{01}$, from the age of the universe. The Reiss et al. measurement methodology is based on evaluating distances from astronomical objects and their receding velocities from earth. This methodology involves looking back in time toward the horizon (edge of the observable universe). Since the measurement never gets to the era of the Big Bang, it cannot include the elapsed time between the Big Bang event and the release of photons.

Also, it is seen that in the evaluation of the Hubble time, $t_{H_{02}}$, as given by equation (30), the light travel time is increased by $\delta t_{02}$ as given in equation (28). But the value of $H_{02}$ given by equation (32) is consistent with the value of $H_{0}=67.66 \pm$ 0.42 obtained by the Plank Collaboration [3]. Thus it is concluded that the Plank Collaboration methodology of estimating the Hubble constant effectively results in the addition of the elapsed time, $\delta t_{02}$, to the age of the universe. The Plank Collaboration estimate of the Hubble constant is based on the considerations of the cosmic microwave background (CMB). In effect, the Plank Collaboration approach involves using the data of the variations of temperature fluctuations together with the Standard $\Lambda$ CDM cosmological model to estimate $H_{0}$. But CMB temperature fluctuations start in the era of the Big Bang. Thus Plank Collaboration measurement methodology involves the era of the Big Bang, resulting in looking forward from that era toward the present time. In this case the measurement methodology effectively adds the elapsed time to the age of the universe.

Thus it is seen that the difference between the two measured values for the Hubble constant is due to subtraction of $\delta t_{01}$ from, and addition of $\delta t_{02}$ to, the present age of the universe, $t_{0}$. And that essentially depends on how the measurement methodology has incorporated the direction of evaluation, that is, toward or away from the time of the Big Bang. It has been shown by Ripalda [12] that "the geometry of space-time measured by a past-pointing observer is not the same as the geometry of space-time measured by a futurepointing observer". Here we have shown that physically $\delta t_{0}$ represents the elapsed time between the event of the Big Bang and the release of photons and that $\delta t_{01}$ and $\delta t_{02}$ are the cause of the difference between the two measured values of $H_{0}$. Thus the tension $[13,14]$ between the Reiss et al. and the Plank Collaboration measurements of the Hubble Constant is resolved. 


\section{Consistency of the Predictions of the Proposed Analytical Model with Observational Data}

We now show the validity of the proposed analytical model by comparing its predictions of the distance moduli with the observational data. Based on equation (12), the luminosity distance $d_{L}$ is given by

$$
d_{L}=c t_{0}(1+z)^{v}(1+2 z)(1-a)
$$

where, to transform the time origin from the time of the big bang to the present time, the scale factor $a$ in equation (12) has been replaced by $(1-a)$. Substituting for $a$ from relation (8) into the above equation and changing the unit of luminosity distance to Megaparsecs $\left(3.08567758149137 \times 10^{22} \mathrm{~m}\right)$ yield

$$
d_{L}=\frac{c t_{0}}{\text { Megaparsec }}(1+z)^{v}\left(\frac{z+2 z^{2}}{1+z}\right)
$$

The distance modulus $\mu$ is defined by

$$
\mu=25+5 \log _{10} d_{L}
$$

Equation (35) is plotted in figures 2 and 3 for the values of redshift, $z$, varying from zero to 10 . To check how well the curve in these figures represents the reality, the following sets of observational data are also plotted in these figures:

1. A set of $557 \mathrm{SNe}$ data with redshifts from a low of $\mathrm{z}=0.0152$ to a maximum of $\mathrm{z}=1.4$, as reported in 2010 in the Union2 Compilation [15]. In figures 2 to 5 these data points are shown in red.

2. A set of 394 extragalactic distances to 349 galaxies at cosmological redshifts significantly higher than the Union2 Compilation with redshifts from a low of $\mathrm{z}=0.133$ to a maximum of $\mathrm{z}=6.6$, as reported in 2008 by Madore and Steer [16]. In figures 2 to 5 these data points are shown in blue.

As seen from figures 2 and 3, the remarkable consistency of the proposed model's predictions with the observational data validates the analytical model. The proposed analytical model does not require the Hubble constant for its prediction. To further validate the proposed model, in the following section, its predictions of distance moduli will be compared with those of the standard $\Lambda \mathrm{CDM}$ model.

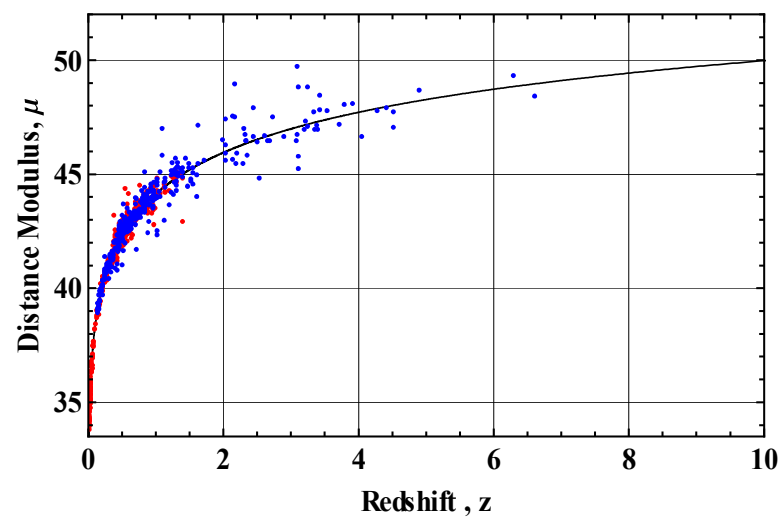

Figure 2. Comparison of the Prediction of the Model with Observational Data.

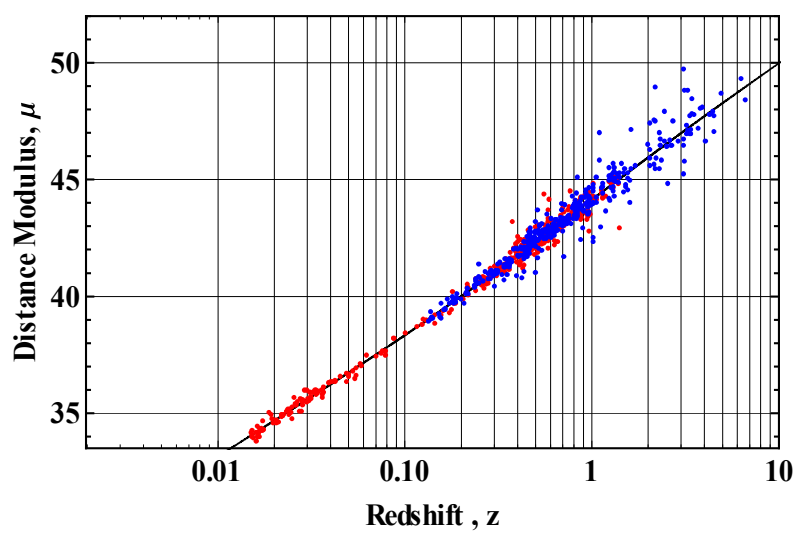

Figure 3. Comparison of the Prediction of the Model with Observational Data.

\section{Consistency of the Predictions of the Proposed Model with Those of the Standard $\Lambda$ CDM Model}

The $\Lambda$ CDM-based models characterize the universe with specific combinations of energy density parameters as fractions of constituent ingredients. The values of these fractions are estimated through finding the optimum fit to the observationally measured data. The results are presented in terms of distance modulus versus redshift. The relation between luminosity distance, $\overline{\overline{\mathrm{d}}}_{L}$, and the redshift is given by the following equation $[7,17,18]$.

$$
\overline{\bar{d}}_{L}=\frac{c}{H_{0}}(1+\mathrm{z}) \int_{0}^{\mathrm{z}} \frac{\mathrm{d} \xi}{\sqrt{\Omega_{\mathrm{m}}(1+\xi)^{3}+\Omega_{\mathrm{r}}(1+\xi)^{4}+\Omega_{\mathrm{k}}(1+\xi)^{2}+\Omega_{\Lambda}}}
$$

where $\mathrm{H}_{0}$ represents the Hubble constant. The quantities $\Omega_{\mathrm{m}}$, $\Omega_{\mathrm{r}}, \Omega_{\mathrm{k}}$, and $\Omega_{\Lambda}$ are the mass, the radiation, the curvature, and the cosmological density parameters. The most recent values for these density parameters as proposed by Reiss et al. (2019) [2] and by the Plank Collaboration (2018) [3] are listed in Table 1 . The distance modulus, $\mu$, is given by

$$
\mu=25+5 \log _{10} \overline{\overline{\mathrm{d}}}_{L}
$$

where the luminosity distance $\overline{\bar{d}}_{L}$ is in Megaparsecs. Using the Plank Collaboration parameters and the Reiss et al. parameters given in Table 1, equation (37) is plotted for both sets of parameters in figures 4 and 5 . The analytical model's prediction, as given by equation (35), and the observational data are also plotted in these figures. Even though some of the Reiss et al. parameters are significantly different from the corresponding parameters of the Plank Collaboration, the Reiss et al. and the Plank Collaboration predictions of distance moduli, as seen in figures 4 and 5, are about the same. This result is not unexpected because each set of parameters in table 1 has been selected such that it would yield the optimum fit to the observational data. These figures also show that the analytical model's prediction lies just in between the Reiss et al. and the Plank Collaboration predictions. This amazing consistency of the curve of the analytical model with the curves of the $\Lambda$ CDM models provides further evidence of the validity of the proposed 
analytical model.

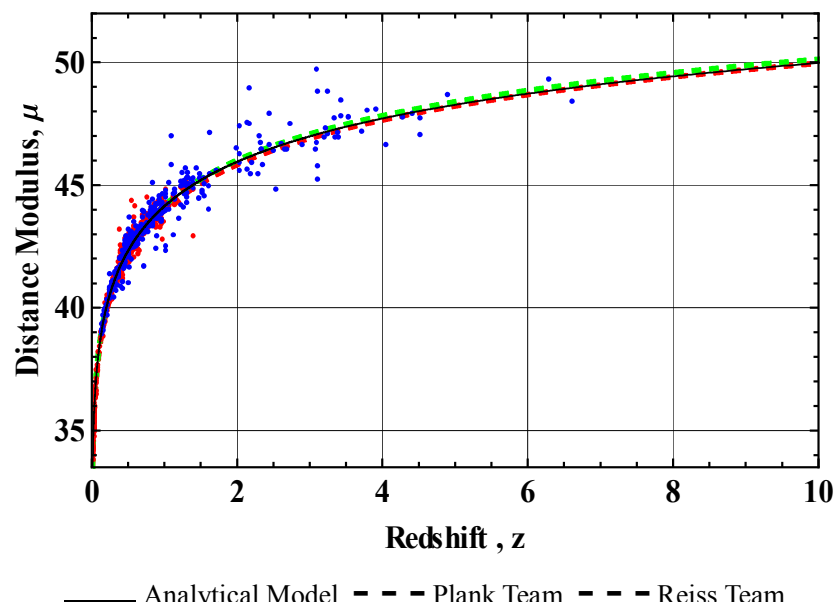

Figure 4. Comparison of the Curve of the Analytical Model with $\Lambda C D M$ Curves.

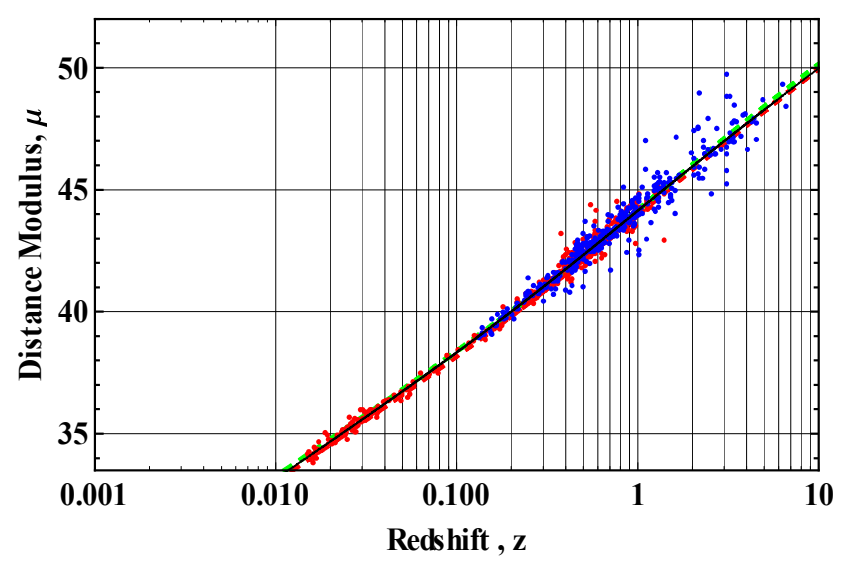

Analytical Model, - - - Plank Team, - - - Reiss Team

Figure 5. Comparison of the Curve of the Analytical Model with $\Lambda C D M$ Curves.

Table 1. Cosmological Parameters for $\Lambda C D M$ Based Models.

\begin{tabular}{lll}
\hline Parameters & Plank Collaboration (2018) & Reiss et al. (2019) \\
\hline $\mathrm{H}_{0}$ & $67.66 \pm 0.42$ & $74.03 \pm 1.42$ \\
$\Omega_{\Lambda}$ & 0.6897 & 0.7 \\
$\Omega_{\mathrm{m}}$ & $1-\left(\Omega_{\Lambda}+\Omega_{\mathrm{r}}+\Omega_{\mathrm{k}}\right)$ & $1-\left(\Omega_{\Lambda}+\Omega_{\mathrm{r}}+\Omega_{\mathrm{k}}\right)$ \\
$\Omega_{\mathrm{r}}$ & 0.0 & 0.0 \\
$\Omega_{\mathrm{k}}$ & 0.0 & 0.0 \\
\hline
\end{tabular}

\section{Conclusion and Remarks}

The proposed analytical model confirms the fidelity of both measured values of the Hubble constant. And it also provides an explanation for the cause of tension between these two well known measured values. Because of the model's remarkable consistency with both the observational data and the standard $\Lambda$ CDM model, it provides a simple tool to estimate the distance moduli without needing the value of the Hubble constant. The model being a kinematic model, does not involve the issues of mass, and energy. The necessary inputs for the proposed model are just the age of the universe and the redshift.

\section{Acknowledgements}

All the numerical work and plots are done via Mathematica v. 10.0, 4/3/2014.

\section{References}

[1] Bull, P., Akrami, Y., Adamek, J., et al., 2016, "Beyond Lambda CDM: Problems, Solutions, and the Road Ahead," "https://arxiv.org/abs/1512.05356.

[2] Riess, A. G., Casertano, S., Yuan, W., et al., 2019, "Large Magellanic Cloud Cepheid Standards Provide a 1\% Foundation for the Determination of the Hubble Constant and Stronger Evidence for Physics Beyond Lambda CDM,": $1903.07603 \mathrm{v} 1 \quad$ [astro-ph.Co] 18 Mar 2019, https://arxiv.org/abs/1903.07603.

[3] Aghanim, N., Akrami, Y., Ashdown, M., et al., Plank 2018 Results, VI. "Cosmological Parameters," arXiv: 1807.06209v1 [astro-ph.Co] 17 Jul 2018.

[4] Freedman, W. L., Madore, B. F., Hatt, D., et al., 2019, “An Independent Determination of the Hubble Constant Based on the Tip of the Red Giant Branch," arXiv: 1907.05922v1 [astro- $\quad$ ph.CO] 12 Jul 2019, https://arxiv.org/abs/1907.05922v1.

[5] NIST, CODATA VALUE: Plank Time, https://physics.nist.gov/cgi-bin/cuu/Value?plkt.

[6] Tian, S., 2017, "The Relation between Cosmological Redshift and Scale Factor for Photons," The Astrophysical Journal, 846-90, 2017 September 10.

[7] David W. Hogg, 2000 Distance Measures in Cosmology, arXiv. astro-ph/9905116v4.

[8] Mukhanov, V., (2005). Physical Foundations of Cosmology. Cambridge University Press.

[9] NIST, CODATA VALUE: Wien Wavelength Displacement Law Constant, http://physics.nist.gov/cgibin/cuu/Value?bwien.

[10] Walker, J., (2008), Fundamentals of Physics, 8th ed., John Wiley and Sons.

[11] Fixen, D. J., 2009, "The Temperature of the Cosmic Microwave Background," The Astrophysical Journal. 707 (2): 916-920. arXiv: 0911.1955 (astro-ph.CO) 10. Nov.

[12] Ripalda, J.M., 2010, “Time Reversal and Negative Energies in General Relativity” arXiv: gr-qc/9906012.

[13] Freeman, W. L., 2017, Cosmology at Crossroads: Tension with the Hubble Constant. https://arXiv.org/abs/1706.02739.

[14] Young, M., 2019, "Tension over Hubble Constant Continues," $\begin{array}{llll}\text { SKY \& Telescope, July 24, } 2019 & 9\end{array}$ https://www.skyandtelescope.com/astronomy-news/tensioncontinues-hubble-constant/.

[15] Amanullah, R., Lidman, C., Rubin, D., Aldering, G., et al., 2010, "Spectra and Hubble Space Telescope Light Curves of Six Types Ia Supernovae at $0.511<\mathrm{z}<1.12$ and the Union2 Compilation," The Astrophysical Journal, 716: 712-738, http://supernova.lbl.gov/Union/figur...n2_mu_vs_z.txt. 
[16] Madore, B. F., Steer, I. P., 2008, "NASA/IPAC Extragalactic Database Master List of Galaxy Distances," NED-4D, http://ned.ipac.caltech.edu/level5/NED4D/.

[17] Peebles, P. J. E., 1993, Principles of Physical Cosmology, Princeton University Press.
[18] Distance Measures in Cosmology - UF Astronomy https://www.astro.ufl.edu/ guzman/ast7939/projects/project01 .html. 